\title{
Distal Aneurysms of Posterior Inferior Cerebellar Artery: Report of Two Cases
}

\author{
Juan L Segura ${ }^{1}$, Hayro Cerna ${ }^{1}$, Yamaurys E Ogando $^{1}$, Rossi E Barrientos ${ }^{1}$ and Aldo Spallone ${ }^{1,2 *}$ \\ ${ }^{1}$ Department of Neurosciences, Faculty of Medicine, Moscow, Russian Federation \\ ${ }^{2}$ Department of Clinical Neurosciences, Neurological Centre of Latium NCL, Italy
}

*Corresponding author: Aldo Spallone, Department of Neurosciences, Faculty of Medicine, Moscow, Russian Federation

\begin{abstract}
ARTICLE INFO
Received: 慧 February 24, 2020

Published: 幽 March 03, 2020

Citation: Juan L S, Hayro C, Yamaurys E O, Rossi E B, Aldo S. Distal Aneurysms of Posterior Inferior Cerebellar Artery: Report of Two Cases. Biomed J Sci \& Tech Res 26(2)2020. BJSTR. MS.ID.004319.
\end{abstract}

Abbreviations: PICA: Posterior Inferior Cerebellar Artery; AVMs: Arteriovenous Malformations; VA: Vertebral Artery; SAH: Subarachnoid Hemorrhage

\begin{abstract}
Background: Aneurysms located in the peripheral segments of Posterior Inferior Cerebellar Artery (PICA) are exceedingly rare. The authors report two cases of distal aneurysms of the PICA which presented hemorrhage and were operated successfully. Unlike many of the reported cases of peripheral PICA aneurysms, both present cases were not associated with other intracranial vascular anomalies. The diagnostic and management aspects of these rare aneurysms are discussed.
\end{abstract}

Methods and Results: Two cases of distal aneurysms of the PICA which presented with hemorrhage and were not associated with any other intracranial vascular anomaly are reported. A midline sub occipital approach and clip occlusion of the lesion was successfully performed in both cases

Conclusion: Although vasospasms and related ischemia are not at all infrequent with these lesions, the results of surgery of these cases are generally good.

Keywords: Aneurysms; Posterior Inferior Cerebellar Artery; Distal Branches; Surgical Treatment

\section{Introduction}

Arterial aneurysms of the PICA are rare. They account for either $1 \%$ or less $[1,2]$ or up to $3 \%$ of all the intracranial aneurysms in different casuistic [3-5]. As rule they are located at the origin of the artery from the vertebral artery. Approximately $15 \%$ of these PICA aneurysms occur distally to the origin of the artery itself [5], a fact which indicates distal PICA aneurysms to be exceedingly rare: 268 of 9709 in Lehto et al. series [5], 60 of 2169 in Peluso et al. series [4], 27 patients from 1989 to 1997 in the Bertalanffy et al. review article [3]

Not infrequently, distal PICA aneurysms can be associated with other lesions, such as posterior fossa AVMs [6], which are likely to play a pathogenic role in the development of these unusual aneurysms. In one of the largest series of PICA aneurysms reported so far [5], 16\% presented hemorrhage from another aneurysm. In this study, 32 of 70 patients harbored multiple aneurysm, and of those 32 patients, 11 presented with a hemorrhage that was not originated from the PICA aneurysm. The present work describes 2 cases of distal PICA aneurysms. Both presented with hemorrhage and were not associated with any other intracranial vascular anomaly. The extreme rarity of these lesions justifies the present report.

\section{Material and Methods}

\section{Case 1}

P.E, 58-year-old women, was admitted to our division after a sudden onset of headache, vomiting and disorientation. Neurological examination revealed nuchal rigidity and confusion. CT scan showed a subarachnoidal hemorrhage associated with an intracerebellar hematoma extending into the 4 tg ventricle. 4-vessel angiography showed an aneurysm of the distal portion 
of the left PICA (Figures 1a \& 1b). One week after admission, the patient underwent operation via a midline sub occipital route. The aneurysm was clipped following extensive anterograde dissection of the artery from its velotonsillary segment to the lesion, which was identified into the contest of the hematoma. Immediately after the clip placement, PICA both proximately and distally to the aneurysm showed a severe vasospasm, which was promptly reversed with topical application of papaverine. Postoperatively, the patient was asymptomatic until the 8th day after surgery, when she suffered headache, dizziness and vomiting just after she stood out of the bed. Neurological examination revealed no deficits. MRI demonstrated a left cerebellar edema, probably due to vasospasm (Figure 2). The patient showed subsequently a gradual improvement. A few days later, postoperative angiogram showed patency of PICA distally to the clipped aneurysm. The patient was discharged 18 days after surgery.
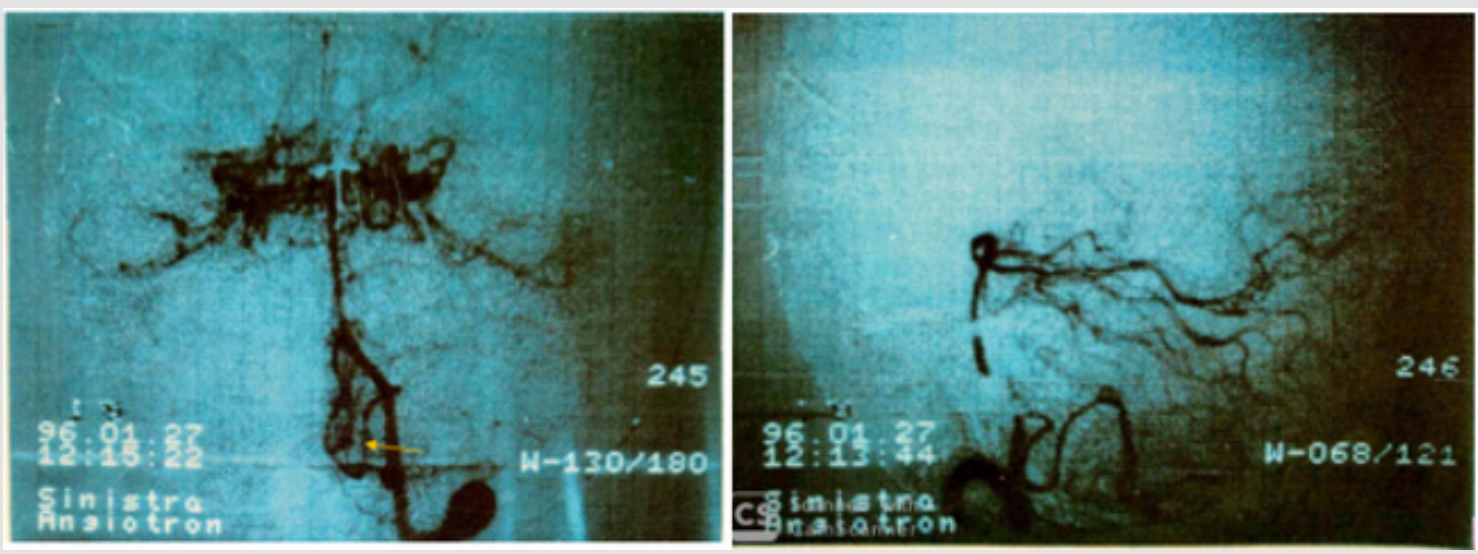

Figure 1:

a) Cerebral angiography, A-P view. This examination gives evidence of an aneurysm located in the velo-tonsillary segment of the left PICA (arrow)

b) Cerebral angiography, A-P view. This examination gives evidence of an aneurysm located in the velo-tonsillary segment of the left PICA (arrow).

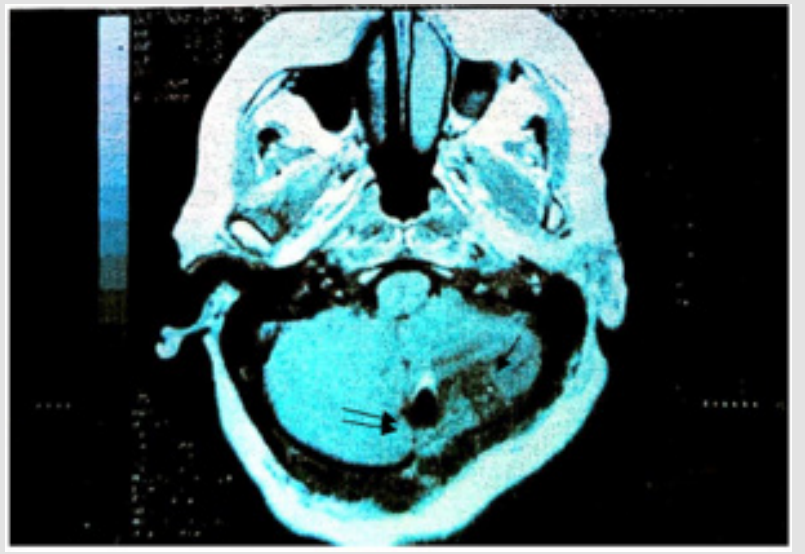

Figure 2: Postoperative MRI gives evidence of a local recent infarct with surrounding edema (arrow). Clip artifact is also shown (double arrows).

\section{Case 2}

P.L. 30-year-old women, was admitted to our division one day after sudden onset of headache and vomiting. Neurological examination revealed mild nuchal rigidity. CT scan showed a subarachnoid hemorrhage located in the upper portion of the vermian cistern as well as the inferior surface of the tentorium. 4 vessel angiographies sowed a distal aneurysm of the left PICA, located just off the midline on the upper vermis. One week after subarachnoid hemorrhage the patient underwent operation by suboccipital approach in the semi sitting position. The $3 \mathrm{~mm}$ aneurysm was identified and clipped following anterograde dissection of the distal PICA up to the vermian segment. Postoperative course was uneventful, and the patient was discharged 14 days after surgery, asymptomatic.

\section{Discussion}

The first case of a PICA aneurysm arising from a distal segment was reported by Fernet in 1864 [7]. These is an extremely 
rare pathology that account for less than 1\% up to $3 \%$ of all intracranial aneurysms in some series [1-5]. Distal PICA aneurysm unassociated with other intracranial anomalies are very rare. They usually present with a subarachnoid and/or intracerebral bleeding not infrequently associated with a fourth ventricle hematoma, because of that, it is not unusual a majority of patients also exhibit hydrocephaly $[8,9]$. Literature from different authors suggested that the incidence in aneurysms arising in association with Arteriovenous Malformations (AVMs) may be as high as 46\% [810]. The size of the aneurysm is an important factor to determine the hemorrhagic risk and the therapeutic options. The "safe size" under which the hemorrhage is less likely to occur is $6 \mathrm{~mm}$ but this does not apply to aneurysms that are located in the distal portion of the PICA because they are small lesions with very thin wall that would harbor a higher risk of rupture [9]. Both present patients harbored small- sized aneurysms, $3 \mathrm{~mm}$ and $5 \mathrm{~mm}$ in diameter respectively, which is the considered minimum size for a cerebral aneurysm to rupture. Bleeding peripheral PICA aneurysms exhibit as a rule a smaller size than other aneurisms [11]. Some series recommend using at least three vessel cerebral arteriography, with selected catheterization of the fourth vessel if there was insufficient reflux of contrast material to visualize the entire contralateral PICA [8].

CT scan represented the main diagnostic tool in our study as well as in most cases reported in the recent literature. Computed tomography demonstrates intracranial blood, whose distribution is related to the actual location of the aneurysm. Bleeding from the proximal PICA segment, including the Vertebral Artery (VA) - PICA junction, usually produces clots within the ipsilateral basal cisterns, with or without extension into the fourth ventricle [12]. On the other hand, lesions arising distal to the medullary PICA segments, mainly those originating from the caudal loop or at a low origin of the PICA are more commonly associated with hemorrhage extending into the fourth ventricle $[12,13]$. In the preset case 2 the blood was detected just under the tentorium and the lesion was located at the bifurcation occurring at the level of the upper vermis. In our case 1 there was an intracerebellar hematoma which extended into de fourth ventricle. This was found to correlate precisely with the location of the bleeding lesion at surgery. MRI was performed in case 1 and matched well with the CT scan data. Post operatively MRI gave evidence of a cerebellar asymptomatic, though significant edema that might have been related to the surgical manipulation and significant intraoperatively - occurring spasm of the PICA.

Angiography represented the main diagnostic tool in the present as well as in the cases reported in the literature. Angiographic vasospasm has been a rather frequent occurrence and more than a half of Subarachnoid Hemorrhage (SAH) patients develop angiographically detectable delayed cerebral vasospasm. This as a rule occurs between 4 and 15 days after ictus and can be associated with neurological deficits that contribute to increased morbidity and mortality after SAH [14]. The goal management of peripheral aneurysms of the PICA is obviously clip occlusion or endovascular obliteration of the lesion with preservation of the lumen of the PICA. If the PICA is sacrificed, leptomeningeal communications with the superior cerebellar artery and the AICA are usually enough to protect against functionally evident cerebellar infarctions [15]. A midline sub occipital approach is adequate for exposure of these lesions. Identification of the appropriate vessel is mandatory in order to proceed to distal dissection and exposure of the lesion; this is usually done by lifting the ipsilateral tonsil and following distally the artery so exposed. An option could be simply to locate the bleeding lesion in the context of a cerebellar/intraventricular hematoma, when present. In the present case 1 we preferred anyway to expose the artery proximally. This required a longer dissection time; however proximal control could be constantly kept. In case 2, this "anterograde" dissection was "conditio sine qua non" for identification and proper exposure of a $3 \mathrm{~mm}$ aneurysm of the vermian branch of PICA. Although surgical treatment of distal PICA aneurysms poses little risk "per se" due to the generally small functional importance of the vessels harboring the lesion, dissection of the parent vessel and proper clipping of the aneurysm is not-atall an easy task due to the small size of the vascular structures in point, that can be easily compromised by an imperfect clipping.

\section{Conclusion}

Peripheral PICA aneurysms are exceedingly rare. Diagnosis requires wise interpretation of $\mathrm{CT}$ and/or MRI data, which usually show a posterior fossa intraparenchimal - intraventricular hemorrhage, and sometimes an unusual distribution of the subarachnoid blood. Local angiographic vasospasm is frequent, and a constant finding is the small size of the bleeding lesion as compared with aneurysms in other locations. Surgical treatment is mandatory and good results of surgery is the rule.

\section{References}

1. Tetsuyoshi Horiuchi, Yuichiro Tanaka, Kazuhiro Hongo, Junpei Nitta, Yoshikazu Kusano (2003) Characteristics of distal posteroinferior cerebellar artery aneurysms. Neurosurgery 53(3): 589-596.

2. Dernbach P, Sila C, Little J (1988) Giant and multiple aneurysms of the distal posterior inferior cerebellar artery. Neurosurgery 22(2): 309-312.

3. Bertalanffy H, Sure U, Petermeyer M, Becker R, Gilsbach J (1998) Management of Aneurysms of the Vertebral Artery-Posterior Inferior Cerebellar Artery Complex. Neurologia medico-chirurgica 38: 93-103.

4. Peluso J, Van Rooij W, Sluzewski M, Beute G, Majoie C (2008) Posterior Inferior Cerebellar Artery Aneurysms: Incidence, Clinical Presentation, and Outcome of Endovascular Treatment. American J of Neuroradiology 29(1): 86-90.

5. Lehto H (2015) Aneurysms of the vertebral and posterior inferior cerebellar arteries. Helsinki University Central Hospital.

6. Schmidt N, Reitz M, Raimund F, Treszl A, Grzuska U (2011) Clinical relevance of associated aneurysms with arteriovenous malformations of the posterior fossa. Neurochirurgica 112: 131-135.

7. (1864) Fernet: Bull Soc Anat Paris 39:495.

8. Lewis S, Jhon D, Peace D, LaFrentz P (2002) Day A Distal posterior inferior cerebellar artery aneurysm: clinical features and management. J Neurosurgery 97(4): 756-766. 
9. Guzman O, Alba G, Molina A (2008) Aneurisma del segmento distal de la arteria cerebelosa posteroinferior: presentación clínica y manejo. Neurociencias 13(4): 261-266.

10. Meisel H, Mansmann U, Alvares H (2000) Cerebral arteriovenous malformations and associated aneurysms: analysis of 305 cases from a series of 663 patients. Neurosurgery 46(4): 793-802.

11. Singh R, Behari S, Kumar V (2012) Posterior inferior cerebellar artery aneurysms: Anatomical variations and surgical strategies. Asian J Neurosurgery 7(1): 2-11.

12. Kallmes D, Lanzino G, Dix J (1997) Patterns of hemorraghe with riptures posterior inferior cerebellar artery aneurysms: CT findings in 44 cases. AJR 169(4): 1169-1171.

ISSN: 2574-1241

DOI: $10.26717 /$ BJSTR.2020.26.004319

Aldo Spallone. Biomed J Sci \& Tech Res

(C) This work is licensed under Creative

Submission Link: https://biomedres.us/submit-manuscript.php
13. Sadato B, Numaguchi Y, Rigamonti D (1991) Bleeding patterns in ruptured posterior fossa aneurysms: a CT study. J Comput Assist Tomogr 15(4): 612-617.

14. Danura H, Schatlo B, Masbacher S (2015) Acute angiographic vasospasm and the incidence of delayed cerebral vasospasm: preliminary results. Acta Neurochi Suppl 120: 187-190.

15. Azzam C (1987) Growth of multiple peripheral high flow aneurysms of the posterior inferior cerebellar artery associated with a cerebellar arteriovenous malformation. Neurosurgery 21(6): 934-939.

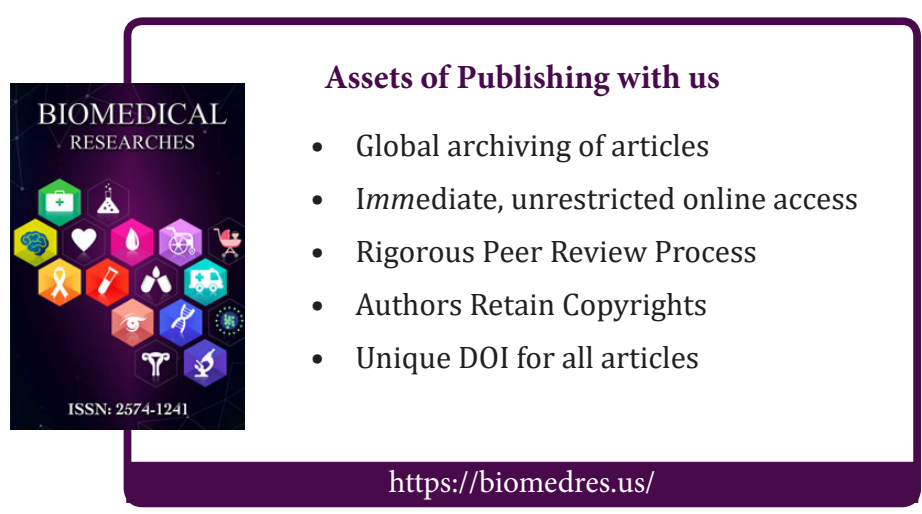

